\title{
ENERGY RESOLUTION AT INTERACTION POINT FOR ASYMMETRIC BEAMS
}

\author{
$\underline{\text { S. Petracca* }}^{*}$ K. Hirata ${ }^{\dagger}$, KEK, Tsukuba, Japan
}

\section{Abstract}

The monochromatic scheme adopted in the tau-charm factories uses a large dispersion at interaction point to obtain a very high collision energy resolution.

Due to the presence of dispersion, however, the beambeam interaction induces the synchro-betatron coupling. Even if in the linear regime, it can lead to potentially serious effects. One of the most serious is the rapid decrease of the energy resolution when the beam-beam parameter increases, even if the motion is stable. In this paper we study this effect in detail either for symmetric and asymmetric beams and give formula for the energy resolution.

\section{INTRODUCTION}

Recently the monochromatization has been considered seriously for future tau-charm factories [1], where a rather large dispersion exists at the IP with opposite signs for both beams. In this case, the dispersion effects can no longer be discussed in the perturbative sense as in the conventional colliders [2, 3]. In a previous paper [4] we discussed the effects of the dispersion at the IP paying enough attention to the mutual interaction between the betatron and the synchrotron degrees of freedom, and the possible problems associated with the monochromatization within the linear approximation of the beam-beam force.

The purpose of the monochromatization is to make the spread $\sigma_{\varepsilon}$ of the collision energy much smaller than the nominal one $\sigma_{\epsilon}^{0}$. Thus this quantity gives the figure of merit of the machine as well as the luminosity. The monochromatic scheme uses beams with opposite dispersion $D$, and realizes a distribution (centered at the IP) in vertical position $y$ analogue (Gaussian) to that one (centered at $E_{0}$ ) in energy $E$. In this way the particles with energy $E_{0}-\epsilon$ collide with those at the same quote in the other beam but with different energy $E_{0}+\epsilon$, then getting as collision energy $E_{C M} \sim 2 E_{0}$.

\section{ENERGY RESOLUTION}

For simplicity we consider the synchrotron motion and one betatron (vertical) oscillation degree of freedom only. The physical variables of a particle for the betatron and synchrotron motions are $\mathbf{x}_{ \pm}=\left(y_{ \pm}, p_{y \pm}, z_{ \pm}, \epsilon_{ \pm}\right)$, where $y_{ \pm}$is the vertical coordinate, $p_{y \pm}$ the vertical momentum normalized by the (constant) momentum $p_{0}$ of the reference particle, $z_{ \pm}$the time advance relative to the reference particle multiplied by the light velocity $c$, and $\varepsilon_{ \pm}=$

\footnotetext{
* On leave of absence from Universita' del Sannio, Benevento and I.N.F.N. Salerno, Italy

$\dagger$ Graduate University for Advanced Studies, Hayama, Japan
}

$\left(E_{ \pm}-E_{0}\right) / E_{0}$ is the energy deviation from the nominal value $E_{0}$ and normalized by it.

In the monochromatization, as well as the luminosity $L$, the spread $\sigma_{w}$ of the collision energy $w-2 E_{0} \equiv \varepsilon_{+}+\varepsilon_{-}$ is important. The luminosity can be expressed as

$$
L=\text { const } \times \int f_{+}\left(y, \varepsilon_{+}\right) f_{-}\left(y, \varepsilon_{-}\right) d y d \varepsilon_{+} d \varepsilon_{-} .
$$

Here $f_{ \pm}\left(y_{ \pm}, \varepsilon_{ \pm}\right)$is the distribution function in the $\left(y_{ \pm}, \varepsilon_{ \pm}\right)$space. If we assume

$$
f_{ \pm}\left(y, \varepsilon_{ \pm}\right)=\frac{\exp \left(\frac{-A_{22}{ }^{ \pm} y^{2}+2 A_{12}{ }^{ \pm} y \varepsilon_{ \pm}-A_{11}{ }^{ \pm} \varepsilon_{ \pm}^{2}}{2 \operatorname{det} A_{ \pm}}\right)}{2 \pi \sqrt{\operatorname{det} A_{ \pm}}},
$$

with $A$ being

$$
A_{ \pm}=\left(\begin{array}{cc}
\left\langle y^{2}\right\rangle_{ \pm} & \langle y \varepsilon\rangle_{ \pm} \\
\langle y \varepsilon\rangle_{ \pm} & \left\langle\varepsilon^{2}\right\rangle_{ \pm}
\end{array}\right)
$$

we get

$$
L=\text { const } \times \frac{1}{\sqrt{2 \pi}} \frac{1}{\sqrt{A_{11}^{+}+A_{11}^{-}}} .
$$

The luminosity density with respect to $w$ is defined as [10]

$$
\Lambda(w)=\frac{1}{L} \int f_{+}\left(y, \varepsilon_{+}\right) f_{-}\left(y, \varepsilon_{-}\right) \delta\left(w-\varepsilon_{+}-\varepsilon_{-}\right) d y d \varepsilon_{+} d \varepsilon_{-} .
$$

Here $w$ stands for the deviation of the collision energy from the nominal one $\left(2 E_{0}\right)$. Then we have

$$
\Lambda(w)=\frac{1}{\sqrt{2 \pi \sigma_{w}^{2}}} \exp \left(-\frac{w^{2}}{2 \sigma_{w}^{2}}\right),
$$

and the formula for the energy resolution for asymmetric beams is

$$
\sigma_{w}^{2}=\frac{\mathcal{A} \operatorname{det} A_{+} \operatorname{det} A_{-}}{A_{11}^{+}+A_{11}^{-}} .
$$

with

$$
\mathcal{A}=\left(\frac{A_{11}^{+}}{d_{+}}+\frac{A_{11}^{-}}{d_{-}}\right)\left(\frac{A_{22}^{+}}{d_{+}}+\frac{A_{22}^{-}}{d_{-}}\right)-\left(\frac{A_{12}^{+}}{d_{+}}-\frac{A_{12}^{-}}{d_{-}}\right)^{2} .
$$

If we assume that two beams are modified symmetrically, we have $A_{11}^{+}=A_{11}^{-}, A_{22}^{+}=A_{22}^{-}$, and $A_{12}^{+}=-A_{12}^{-}$, $A_{ \pm}=A$ and the formula for the energy resolution becomes

$$
\sigma_{w}^{2}=\frac{2 \operatorname{det} A}{A_{11}} .
$$

In the absence of collision $\left(\xi_{0} \simeq 0\right)$ we can assume the following:

$$
A_{ \pm}=\left(\begin{array}{cc}
\beta_{y}^{0} \epsilon_{y}^{0}+D_{0}^{2}\left(\sigma_{\varepsilon}^{0}\right)^{2} & \pm D_{0}\left(\sigma_{\varepsilon}^{0}\right)^{2} \\
\pm D_{0}\left(\sigma_{\varepsilon}^{0}\right)^{2} & \left(\sigma_{\varepsilon}^{0}\right)^{2}
\end{array}\right)
$$


which implies [10]

$$
\sigma_{w}^{2}=\frac{2\left(\sigma_{\varepsilon}^{0}\right)^{2}}{1+\frac{D_{0}^{2}\left(\sigma_{\varepsilon}^{0}\right)^{2}}{\beta_{y}^{0} \epsilon_{y}^{0}}} .
$$

\section{EQUILIBRIUM ENVELOPE}

The equilibrium value of the envelope matrix $\sigma$, where

$$
\sigma_{i j}=\left\langle\left(x_{i}-\bar{x}_{i}\right)\left(x_{j}-\bar{x}_{j}\right)\right\rangle,
$$

is determined by the following equation [9]:

$$
\sigma=M_{b b}^{1 / 2}\left[\bar{\Lambda} M_{a r c} \sigma\left(\bar{\Lambda} M_{a r c}\right)^{t}+\left(I-\bar{\Lambda}^{2}\right) \bar{E}\right]\left(M_{b b}^{t}\right)^{1 / 2},
$$

where

$$
\begin{gathered}
\bar{\Lambda}=H_{0} B_{0} \Lambda\left(H_{0} B_{0}\right)^{-1}, \quad \bar{E}=H_{0} B_{0} E\left(H_{0} B_{0}\right)^{t}, \\
\Lambda=\operatorname{diag}\left(\lambda_{y}, \lambda_{y}, 1, \lambda_{z}^{2}\right), \quad E=\operatorname{diag}\left(\epsilon_{y}^{0}, \epsilon_{y}^{0}, \epsilon_{z}^{0}, \epsilon_{z}^{0}\right) .
\end{gathered}
$$

$\lambda_{y, z}=\exp \left(1 / T_{y, z}\right)$ being the damping constants and $\epsilon_{y, z}^{0}$ the nominal emittances. The one turn matrix from IP $(s=$ $0)$ to IP is [5]:

$$
M=M_{b b}^{1 / 2} M_{a r c} M_{b b}^{1 / 2}
$$

where:

$$
\begin{gathered}
M_{a r c}=M\left(0_{-}, 0_{+}\right)=H_{0} B_{0} \hat{M}_{a r c} B_{0}^{-1} H_{0}^{-1}, \\
\hat{M}_{a r c}=\operatorname{diag}\left(r\left(\mu_{y}^{0}\right), r\left(\mu_{z}^{0}\right)\right), \quad B_{0}=\operatorname{diag}\left(b_{y}^{0}, b_{z}^{0}\right),
\end{gathered}
$$

with

$$
\begin{gathered}
r\left(\mu_{y, z}^{0}\right)=\left(\begin{array}{cc}
\cos \mu_{y, z}^{0} & \sin \mu_{y, z}^{0} \\
-\sin \mu_{y, z}^{0} & \cos \mu_{y, z}^{0}
\end{array}\right), \\
b_{y, z}^{0}=\operatorname{diag}\left(\sqrt{\beta_{y, z}^{0}}, 1 / \sqrt{\beta_{y, z}^{0}}\right), \\
H_{0}=\left(\begin{array}{cc}
I & h_{0} \\
h_{0} & I
\end{array}\right), \quad h_{0}=\left(\begin{array}{cc}
0 & D_{0} \\
0 & 0
\end{array}\right) .
\end{gathered}
$$

$\mu^{0}=2 \pi \nu^{0}, \nu^{0}$ being the nominal tune, $\beta_{y, z}^{0}$ the nominal betatron functions at IP $\left(\beta_{z}^{0} \equiv \sigma_{z}^{0} / \sigma_{\varepsilon}^{0}, \sigma_{z}^{0}\right.$ being the nominal bunch length), and $D_{0}$ the dispersion at IP. Note that $H_{0}, B_{0}$, and $\hat{M}_{a r c}$ are symplectic. Finally the beam-beam interaction is described as a linear kick

$$
M_{b b}=\left(\begin{array}{cccc}
1 & 0 & 0 & 0 \\
-4 \pi \xi_{0} / \beta_{y}^{0} & 1 & 0 & 0 \\
0 & 0 & 1 & 0 \\
0 & 0 & 0 & 1
\end{array}\right)
$$

with $\xi_{0}$ being the vertical (nominal) beam-beam parameter

$$
\xi_{0}=\frac{2 \pi \gamma r_{e} N \beta_{y}^{0}}{2 \pi \gamma \sigma_{y}^{0}\left(\sigma_{y}^{0}+\sigma_{x}^{0}\right)} .
$$

Note that the nominal synchrotron tune $\nu_{z}^{0}$ is negative for conventional electron machines with positive momentum compaction factor $\alpha_{p}$, and we have assumed that there is only one IP which is a symmetric point with respect to betatron and synchrotron motions. We have also implicitly assumed that dispersion does not exist in cavities.

\section{DISCUSSIONS}

In Fig. 1 we show that the energy resolution $\sigma_{w}$ increases rapidly with $\xi_{0}$ and approaches its nominal value $\sigma_{\epsilon}^{0}$, then making the monochromatization less effective or even useless. This, obviously, gives a more stringent limit for the maximum value of $\xi_{0}$ than the single particle instability [4].

Furthermore, from Fig. 1, it is clear that this effect weakly depends on the betatron tunes and is almost indipendent on the synchrotron ones.

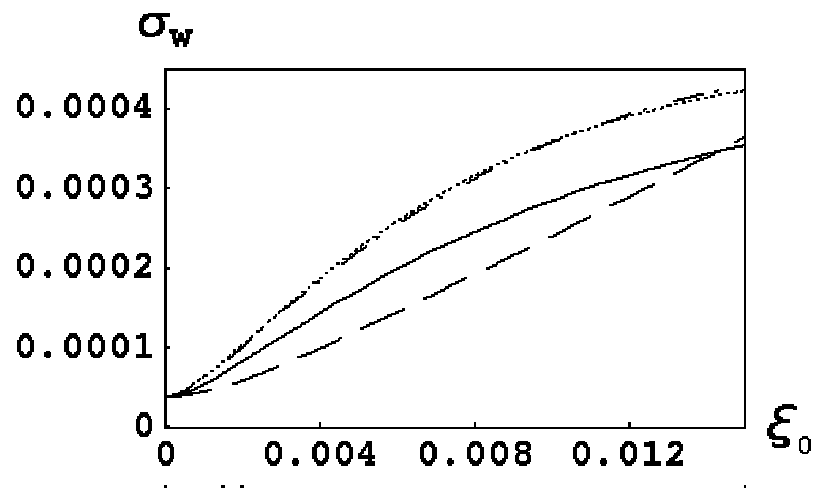

Figure 1: The energy resolution $\sigma_{w}$ versus $\xi_{0}$ with $D_{0}=$ $0.4 \mathrm{~m}$ and other parameters are in Table 2 .

Table 1: Legenda of Figure 1

\begin{tabular}{|l|c|c|c|c|c|}
\hline line & $\nu_{y}^{0+}$ & $\nu_{y}^{0-}$ & $\nu_{z}^{0+}$ & $\nu_{z}^{0-}$ & beams \\
\hline dash-dot & 0.05 & 0.05 & 0.03 & -0.03 & asymmetric \\
dash & 0.1 & 0.05 & 0.03 & -0.08 & asymmetric \\
solid & 0.1 & 0.05 & 0.03 & 0.03 & asymmetric \\
dot & 0.05 & 0.05 & 0.03 & 0.03 & symmetric \\
\hline
\end{tabular}

Table 2: Parameters for both beams

\begin{tabular}{|c|c|c|c|}
\hline$\beta_{y}^{0}$ & $0.03 \mathrm{~m}$ & $\beta_{z}^{0}$ & $26.3 \mathrm{~m}$ \\
$\epsilon_{y}^{0}$ & $410^{-9} \mathrm{~m}$ & $\epsilon_{z}^{0}$ & $3.810^{-6} \mathrm{~m}$ \\
$\sigma_{\varepsilon}^{0}$ & $3.810^{-4}$ & $\sigma_{z}^{0}$ & $0.01 \mathrm{~m}$ \\
$T_{y}$ & 1000 & $T_{z}$ & 500 \\
\hline
\end{tabular}

The reason of the rapid growth of the energy resolution with $\xi_{0}$ is due to one of $\sigma_{11}$ and in particular of $\sigma_{22}$ [8], hence of the vertical and longitudinal emittances respectively. They increase in a similar manner regardless of the sign of $\nu_{z}^{0}$, even if for negative $\nu_{z}^{0}$ the increase might be easier to understand, because the system is unstable [4].

The emittances are obtained from the envelope matrix $\sigma$ as follows:

$$
\text { Eigenvalues }[J \sigma]=\left\{i \epsilon_{y},-i \epsilon_{y}, i \epsilon_{z},-i \epsilon_{z}\right\} .
$$

In Fig. 2 we plot the emittances $\epsilon_{y, z}$ as functions of $\xi_{0}$, for $\nu_{z}^{0}=0.08$. This shows that the longitudinal emittance $\epsilon_{z}$ is 
influenced considerably by the the beam-beam force. This effect has been usually overlooked in the literature where the synchrotron oscillation is assumed to be unaffected. Also, the vertical emittance $\epsilon_{y}$ grows up quite rapidly.
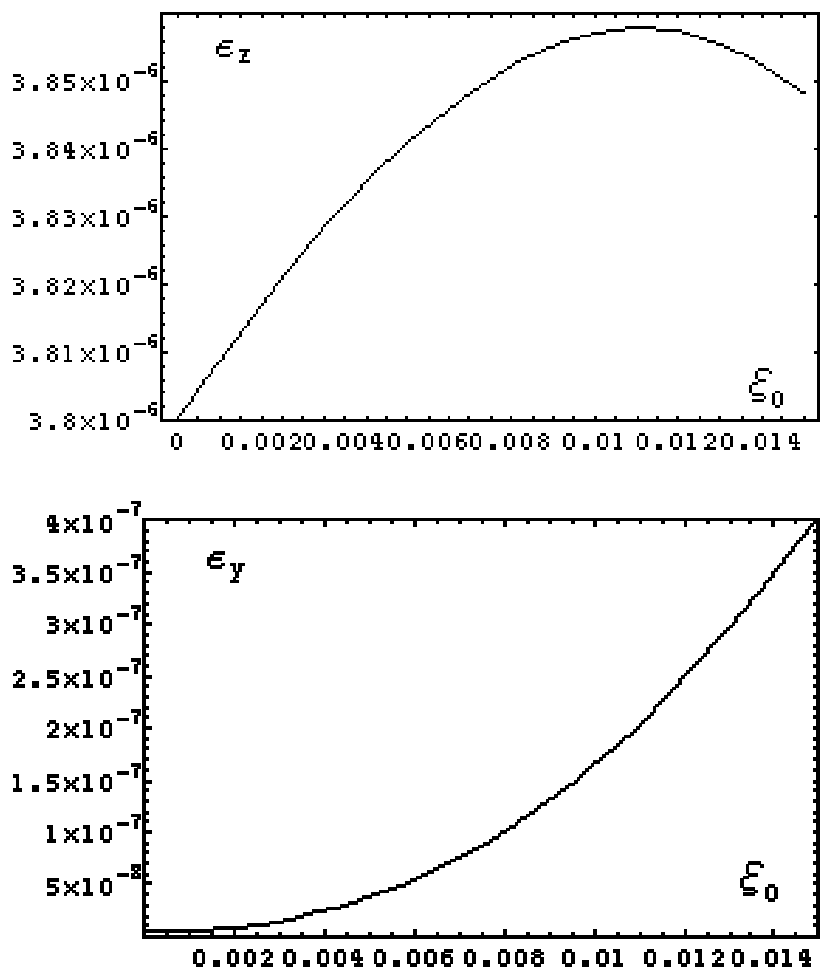

Figure 2: The synchrotron emittance $\epsilon_{z}$ (top) and the betatron one $\epsilon_{y}$ (bottom) as functions of $\xi_{0}$ with $\nu_{z 0}=0.08$ and all other parameters are listed in Table 2.

As conclusion, within the linear analysis used in this paper, it seems difficult to avoid this dangerous effect. However there can be some other effects in nonlinear regime which is worth to study. In deciding the machine parameters one should pay enough attention to the energy resolution.

\section{REFERENCES}

[1] IHEP-BTCF Report-01, Dec. (1995) (unpublished); Yu. I. Alexahin A. N. Dubrovin and A. A. Zholents, Proc. of 2nd EPAC, 398 (1990); A. A. Zholents, AIP Conf. Proc. 214, 592 (1990); A. Faus-Golfe and J. Le Duff, Nucl. Inst. Meth. A 372, 6 (1996); P. F. Beloshitsky, JINR, EP-92-187, Dubna (1992)(unpublished); J. M. Jowett, Frontiers of Particle Beams: Factories with e+e-Rings, eds. M. Denies, M. Month, B. Strasser, S. Turner, Springer Verlag (1994), and references given there.

[2] A. Renieri, INF-75/6(R), February 1975. A. Piwinski, DESY 77/18, 1977, and DESY H1/71-1, (1971) (unpublished).

[3] Y. Kamiya and A. W. Chao, SLAC/AP-10, (1983) (unpublished).
[4] S. Petracca and K. Hirata, Phys. Rev. D59, 1, R 40 (1999).

[5] K. Ohmi, K. Hirata and K. Oide, Phys. Rev. E-49, 751 (1994).

[6] S. X. Fang et al. Part.Acc. 51, 15 (1995).

[7] K. Hirata, H. Moshammer and F. Ruggiero, Part. Acc. 40, 205 (1993).

[8] S. Petracca and K. Hirata, KEK Preprint 97-28 (1996).

[9] K. Hirata and F. Ruggiero, Part. Acc., 28, 137 (1990).

[10] M. Bassetti and J. M. Jowett, IEEE Proc. of the PAC 1987, 115 (1987). 